\title{
DYNAMICS AND CONTROL OF NONHOLONOMIC MOBILE ROBOT SYSTEMS
}

\author{
W. SCHIEHLEN \\ Institute B of Mechanics, University of Stuttgart, D-70550 Stuttgart, Germany
}

\begin{abstract}
The positional degrees of freedom of a mobile robot are reduced by nonholonomic constraints further to a smaller number of motional degrees of freedom. It is shown how the equations of motion can be reduced to a minimal number using generalized coordinates and generalized velocities. The theoretical results are applied to an actively controlled robot with stiff tires. One scalar control variable provides full contollability of the position of the robot moving on a plane surface. A control strategy is found for stationary and instationary motions.
\end{abstract}

Key Words. Computer simulation; modeling; nonlinear control system; robots; system order reduction; nonholonomic systems

\section{INTRODUCTION}

Nonholonomic systems have been studied in dynamics for a long time, see Neimark and Futaev (1972). In principle, the equations of motion may be obtained by two approaches: i) application of D'Alembert's principle or Lagrange's equations of the second kind for the evaluation of the equations considering the holonomic constraints only and adding Lagrangian multipliers to the equations to represent the nonholonomic constraints or ii) application of Jourdain's principle considering the nonholonomic constraints immediately. Jourdain's principle, see e. g. Bahar (1993), Schiehlen (1986), also referred to as Kane's equations, see e. g. Kane (1978), results in a minimal number of ordinary differential equations (ODEs) while the Lagrangian multiplier approach leads to a maximal number of differential algebraical equations (DAEs). However, the Lagrangian multipliers may be eliminated using the nonholonomic constraints as shown, e. g., by Wittenburg (1977).

More recently nonholonomic systems found increasing interest in applied mathematics. Geometrical methods proved to be most efficient in modeling and analysing mechanical systems, Marsden et al. (1990), Krish- naprasad et al. (1991), Yang (1992). In particular the reduction of the equations of motion to minimal Riemannian spaces is an important topic.

In this paper, the reduction of nonholonomic systems will be treated from a mechanical point of view. Then, the motion of a nonlinearily controlled robot with nonholonomic constraints will be discussed in detail. Simulation results present an illustrative overview on the dynamical behaviour of such a robot. In particular, it is shown that the plane motion of a robot with three degrees of freedom can be completely controlled by one control variable only.

\section{EQUATIONS OF MOTION}

Nonholonomic systems like skaters and service robots can be modeled properly as multibody systems for dynamical analysis. The theoretical background is today available from a number of textbooks authored e. g. by Roberson and Schwertassek (1988), Nikravesh (1988), Haug (1989) and Shabana (1989). The state - of - the-art is also presented at a series of IUTAM/IAVSD symposia and NATO Advanced Study Institutes, documented in the corresponding proceedings, see, e. g., Magnus (1978), Haug (1984), Bianchi and Schiehlen 
(1986), Kortüm and Sharp (1993), Haug (1993).

The method of multibody systems is based on a finite set of elements such as rigid bodies and/or particles, bearings, joints and supports, springs and dampers, active force and/or position actuators.

The multibody system model has to be described mathematically by equations of motions. For control purposes a minimal number of generalized coordinates and velocities is most perferable, in particular for nonholonomic systems like mobile robots. A summary of such a modeling approach was presented $\mathrm{e}$. g. by Schiehlen (1993).

\section{NONLINEARLY CONTROLLED NON- HOLONOMIC ROBOT}

The state equations of a four-wheeled robot, Fig. 1, are generated based on the following assumptions.

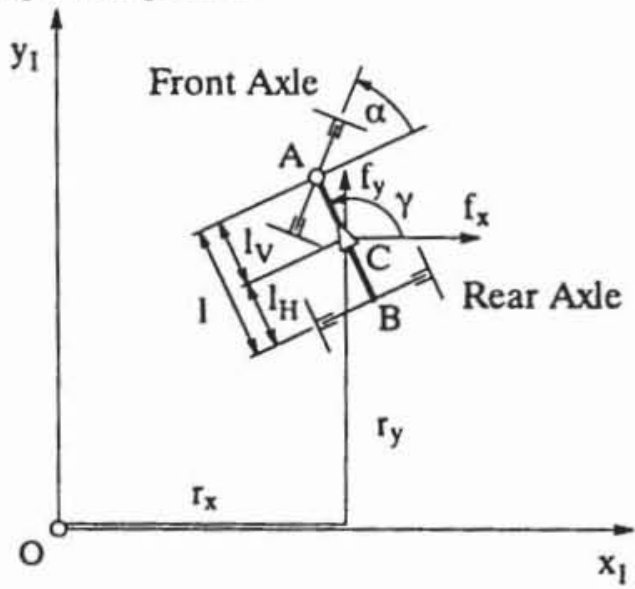

Fig. 1: Robot model

1. The robot is considered as one rigid body. Front axle and rear axle including the wheels are considered as massless.

2. Steering follows from rotation of the whole front axle.

3. The wheels roll on a rough plane. Sliding or loss of contact are excluded.

4. The drive force acts always on the center of gravity $C$ of the robot.

The rough plane the robot is rolling on is described by fixed axes $x_{b}, y_{l}$. The robot position within the plane is defined by coordinates $r_{x}, r_{y}$ of the center of gravity $C$ and angle $\gamma$ due to the robot longitudinal axes. Thus, the robot has three positional degrees of freedom. However, the velocity degrees of freedom of the robot are constrained by the rolling condition of the massless but rigid axles. The instantaneous velocities $v_{A}, v_{B}$ of the points $A$ and $B$ are vanishing in the directions of the axles leading to the implicit contraints

$$
\begin{aligned}
& v_{A} \equiv\left(\dot{r}_{x} \cos \gamma+\dot{r}_{y} \sin \gamma\right) \tan \alpha-l \dot{\gamma}=0, \\
& v_{B} \equiv-\dot{r}_{x} \sin \gamma+r_{y} \cos \gamma-l_{H} \dot{\gamma}=0 .
\end{aligned}
$$

The constraints (1) and (2) are linear, nonholonomic and rheonomic, if the steering angle $a(t)$ is any function of time. The constraints may be expressed explicitly, too,

$\left.\begin{array}{l}\dot{r}_{x}=v_{H}\left[\cos \gamma-\left(l_{H} / l\right) \tan a \sin \gamma\right], \\ \dot{r}_{y}=v_{H}\left[\sin \gamma+\left(l_{H} / l\right) \tan a \cos \gamma\right] \\ \dot{\gamma}=v_{H}(1 / l) \tan \alpha,\end{array}\right\}$

where the velocity of the rear axle,

$v_{H}=\dot{r}_{x} \cos \gamma+\dot{r}_{y} \sin \gamma$,

was introduced as abbreviation. Thus, the robot has one degree of freedom with respect to the velocity, described definitely by the generalized velocity $v_{H}$

The free control variables of the robot are at first given by the steering angle $\alpha(t)$ and the coordinates $f_{x}(t), f_{y}(t)$ of the driving force. Then, supplying the robot with a velocity dependent steering control, which is following the law

$a=\arctan \left(v_{H} / v_{L}\right)$,

leads to a loss of one free control variable, and the robot motion remains controlled only by the driving forces $f_{x}(t), f_{y}(t)$. Here, $v_{L}$ is a reference velocity.

Together with (5), constraint (1) reads now

$v_{A} \equiv\left(\dot{r}_{x} \cos \gamma+\dot{r}_{y} \sin \gamma\right)^{2}-v_{L} l \dot{\gamma}=0$.

Hence, the robot is governed by a nonlinear, nonholonomic and skleronomic constraint, and a nonlinear, nonholonomic system is given. The explicit form of the constraints are changed correspondingly,

$\begin{aligned} & \left.\dot{r}_{x}=v_{H} \cos \gamma-\left(v_{H}^{2} l_{H} / v_{L} l\right) \sin \gamma\right], \\ & \left.\dot{r}_{y}=v_{H} \sin \gamma+\left(v_{H}^{2} l_{H} / v_{L} l\right) \cos \gamma\right] \\ & \dot{\gamma}=v_{H}^{2} / v_{L} l,\end{aligned},$.

The number of degrees of freedom with respect to the position and the velocity will not be changed by the steering control.

The equation of motion will be generated by Jourdain's principle, see e. g. Schiehlen (1986). According to that, for the plain motion of a rigid body one gets

$\left(m \ddot{r}_{x}-f_{x}\right) \delta^{\prime} \dot{r}_{x}+\left(m \ddot{r}_{y}-f_{y}\right) \delta^{\prime} \dot{r}_{y}+I \ddot{\gamma} \delta^{\prime} \dot{\gamma}=0$ 
where $m$ is the robot mass and $I$ the moment of inertia with respect to $C$. One gets the accelerations directly by differentiating (7), while Jourdain's variations $\delta$ ' $\dot{r}_{x} \delta \dot{r}_{y}, \delta^{\prime} \dot{\gamma}$ have to be derived from (3) and (5), since $\delta^{\prime} t=0$. After some lenghty calculations, from (8) one gets the equation of motion

$$
\begin{aligned}
{[m+} & \left.2\left(I+m l_{H}^{2}\right) \frac{v_{H}^{2}}{v_{L}^{2} l^{2}}\right] \dot{v}_{H} \\
= & f_{x}\left(\cos \gamma-\frac{v_{H} l_{H}}{v_{L} l} \sin \gamma\right) \\
& +f_{y}\left(\sin \gamma+\frac{v_{H} l_{H}}{v_{L} l} \cos \gamma\right) .
\end{aligned}
$$

The robot state is completely defined by the coupled nonlinear system of differential equations (7) and (9). Using the dimensionless quantities

$x=\frac{r_{x}}{l}, y=\frac{r_{y}}{l}, \quad v=\frac{v_{H}}{v_{L}}$,

$u=\frac{l f}{m v_{L}^{2}}, \tau=\frac{v_{L} t}{l}$

one gets dimensionless state equations

$$
\begin{aligned}
x^{\prime} & =v \cos \gamma-v^{2} x \sin \gamma, \\
y^{\prime} & =v \sin \gamma+v^{2} x \cos \gamma, \\
\gamma^{\prime} & =v^{2}, \\
{[1} & \left.+2\left(i^{2}+x^{2}\right) v^{2}\right] v^{\prime}=u_{x}(\cos \gamma-v x \sin \gamma) \\
& +u_{y}(\sin \gamma+v x \cos \gamma),
\end{aligned}
$$

where the abbreviations $x=l_{H} / l$ and $i^{2}=I / m l^{2}$ have been introduced.

\section{DYNAMICAL ANALYSIS OF MOTION}

Nonholonomic motion planning is a nontrivial task, see e. g. Zexiang $\mathrm{Li}$ and J. F. Canny (1993). In particular, a vehicle with only one control input requires a thorough dynamical analysis. Therefore, stationary and instationary motion of the robot will be considered, see also Schiehlen (1977). A further reduction is obtained for motion under gravity only. The resulting conservative system, or system with symmetry, respectively, has an energy integral omitting one differential equation.

\subsection{Stationary Motion}

Without drive,

$u_{x}=u_{y}=0$, the robot performs a stationary motion. Directly from (11.3) and (11.4) one gets

$v=v_{0}=$ const ,

$\gamma=\gamma_{0}+v_{0}^{2} \tau$.

In addition, from (11.1) and (11.2) follow by integration

$$
\begin{aligned}
& x=x_{m}+x \cos \gamma+\left(1 / v_{0}\right) \sin \gamma, \\
& y=y_{m}+x \sin \gamma-\left(1 / v_{0}\right) \cos \gamma .
\end{aligned}
$$

Thus, the center of gravity is moving on a circular path,

$$
\left(x-x_{m}\right)^{2}+\left(y-y_{m}\right)^{2}=x^{2}+\left(1 / v_{0}\right)^{2}=a^{2}
$$

Due to the steering law (5), the robot can't perform a stationary straight motion. The radius $a$ of the circular path is decreasing with increasing initial velocity $v_{0}$. The circle center has the coordinates

$$
\left.\begin{array}{l}
x_{m}=x_{0}+x \cos \gamma_{0}-\left(1 / v_{0}\right) \sin \gamma_{0} \\
y_{m}=y_{0}-x \sin \gamma_{0}+\left(1 / \nu_{0}\right) \cos \gamma_{0}
\end{array},\right\}
$$

defined by the initial conditions $x_{0}, y_{0}$ and $\gamma_{0}$ at time $\tau_{0}$.

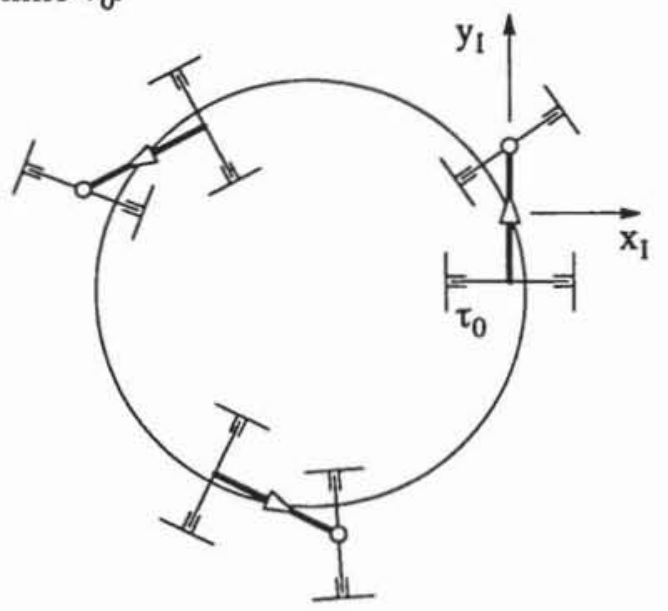

Fig. 2: Stationary circular motion

Fig. 2 shows the stationary motion of the circular motion with $v_{0}=0,8$. The radius is $a=1,346$, and the period time reads $T=2 \pi / \nu_{0}^{2}=9,817$.

\subsection{Instationary Motion}

The robot with drive performs instationary motions. Here, the most important cases of rear axle drive and of running down an inclined plane will be treated.

The rear axle drive is characterized by a force acting always in the direction of the longitudinal robot axes:

$u_{x}=u \cos \gamma, u_{y}=u \sin \gamma$, 
where $u$ represents the value of the driving force. Then it follows from (11.4) the equation

$\left[1+2\left(i^{2}+x^{2}\right) v^{2}\right] v^{\prime}=u(t)$

with one free control variable $u(t)$ only. Equation (11.1) to (11.3) remain unchanged.

The differential equation (20) might be integrated directly. The result is a cubic equation for the velocity,

$\frac{2}{3}\left(i^{2}+x^{2}\right) v^{3}+v$

$-\left[\frac{2}{3}\left(i^{2}+x^{2}\right) v_{0}^{3}+v_{0}+\int_{\tau_{0}}^{\tau} u d \sigma\right]=0$.

This cubic equation can be solved elementarily, but this isn't very profitable, because in a general case equations (11.1) to (11.3) can be integrated only numerically.

Fig. 3 shows the accelerated robot with rear axle drive starting from a rest. One recognizes the parabolic curve due to small velocities near the origin, while the circular path with decreasing radius appears distinctly in the case of high volocities. In Fig. 4 appears a peak in the trajectory for the decelerated motion. The peak refers to a change in direction of the robot with a momentary rest position.

$$
\begin{aligned}
& \mathrm{v}_{0}=0 \\
& \mathrm{u}=0,1
\end{aligned}
$$

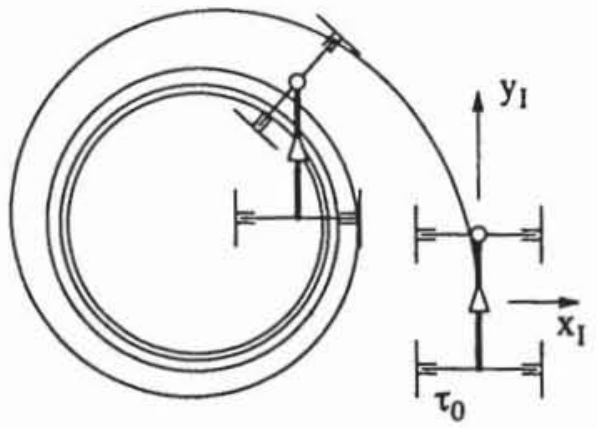

Fig. 3: Constant acceleration starting at a rest position

Destined motions of the robot can be achieved by using a Bang-Bang control of the rear axle drive, i. e., by a single control variable. However, the control system design requires special attention. The control laws have to be designed due to the nonlinearity of the system by parameter studies, but they may be determined graphically, as well. The control

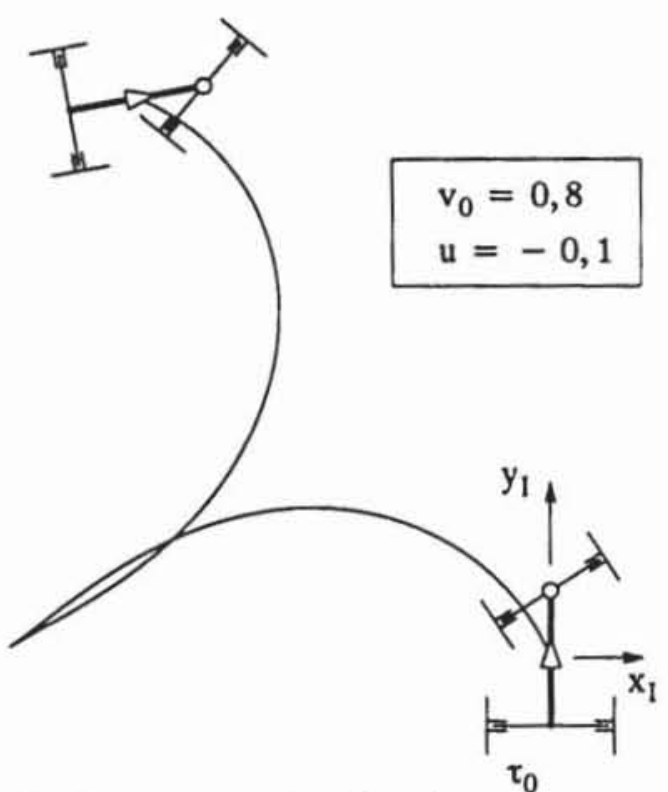

Fig. 4: Constant deceleration from circular motion

laws for the displacement maneuver and the turning maneuver are put together in Tab. 1. Fig. 5 shows the corresponding trajectories. The displacement of the robot can be realized only by circular curves. The turn maneuver can be achieved by a common change in direction. With the change in direction there appears the characteristic trajectory peak, again.

\subsection{Motion Under Gravity}

The inclined plane is rotated relative to the horizontal with respect to the $x$-axes by the angle $\delta$. Then, the applied drive force is always directed parallel to the $y$-axes,

$u_{x}=0, \quad u_{y}=h=g l\left(1 / v_{L}\right)^{2} \sin \delta$,

where $g$ denotes the gravity. Then, from (11.4) follows the equation

$\left[1+2\left(i^{2}+x^{2}\right) v^{2}\right] v^{\prime}=h(\sin \gamma+v x \cos \gamma)$

The state equations (11.3) and (23) now are coupled and can't solved separately any longer. However, the number of state equations might be diminished by the energy integral. Based on vanishing initial conditions, $y_{0}=v_{0}=0$, one finds from (11.2) and (23) the integral

$\left(i^{2}+x^{2}\right) v^{4}+v^{2}-2 h y=0$.

There remain the differential equations (11.1) to (11.3) as state equations. Thus, the numerical integration of the general solution is simplified. 
Displacement maneuver

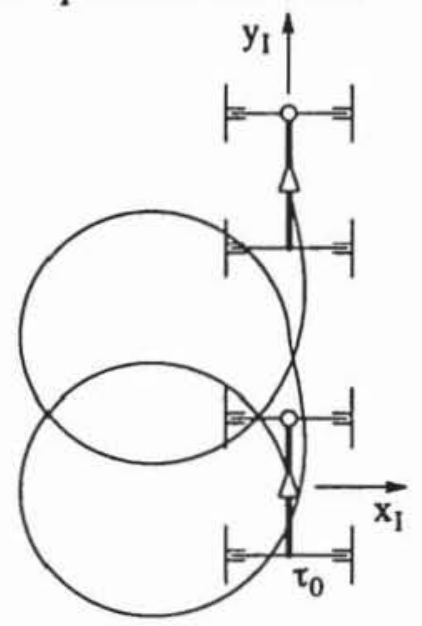

Turning maneuver

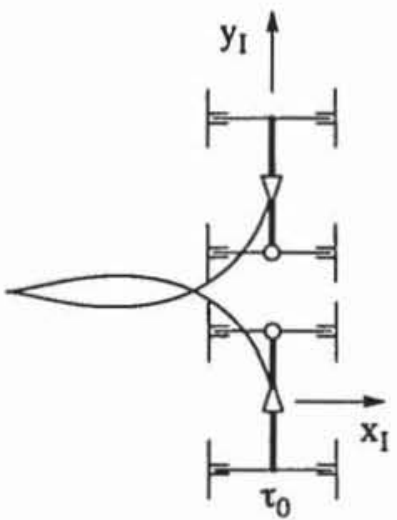

Fig. 5: Bang-Bang control

\begin{tabular}{|c|c|c|c|}
\hline \multicolumn{2}{|c|}{ Displacement maneuver } & \multicolumn{3}{c|}{ Turning maneuver } \\
\hline Time interval & $\begin{array}{c}\text { Control } \\
\text { variable }\end{array}$ & Time interval & $\begin{array}{c}\text { Control } \\
\text { variable }\end{array}$ \\
\hline $0=\tau_{0}<\tau<2,661$ & $\mathrm{u}=+1$ & $0=\tau_{0} \quad<\tau<1,500$ & $\mathrm{u}=+1$ \\
\hline $2,661<\tau<5,322$ & $\mathrm{u}=-1$ & $1,500 \quad<\tau<4,500$ & $\mathrm{u}=-1$ \\
\hline $5,322<\tau<7,983$ & $\mathrm{u}=+1$ & $4,500 \quad<\tau<6,000$ & $\mathrm{u}=+1$ \\
\hline $7,983<\tau<10,644$ & $\mathrm{u}=-1$ & \multicolumn{3}{|c|}{} \\
\hline
\end{tabular}

Table 1: Control laws

On the inclined plane the robot running downwards performs a change in direction as shown in Fig. 6. Thereby, the center of gravity reaches the starting height in a rest position. Then, the robot starts again to run down the in- clined plane, while a mean displacement slanting to the inclined plane takes place. With increasing loss of height the velocity increases, too, and the steering angle grows.

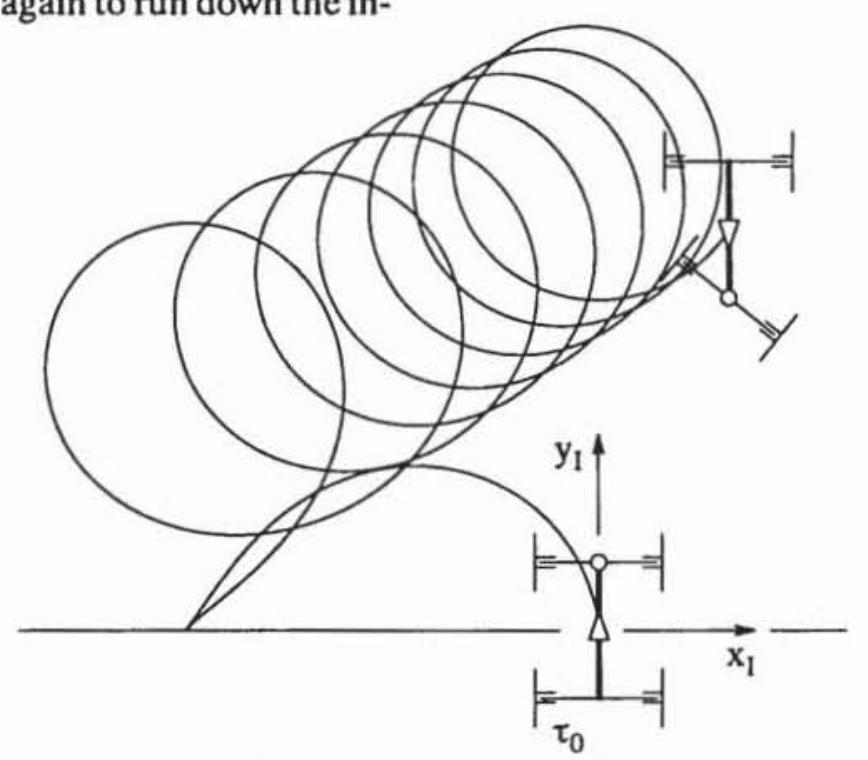

Fig. 6: Running down an inclined plane, $h=0,5$

Fig. 7 shows an interesting special case. The robot starts slanting to the plane from a rest and reaches after changing the direction a horizontal, stationary rest, again. The robot's motion is self-restrained. 


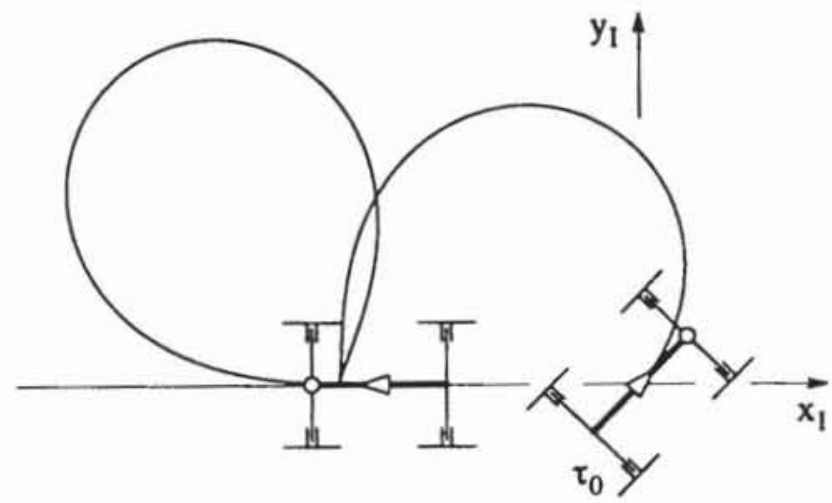

Fig. 7: Self-restraining on the inclined plane, $h=0,5$

\section{CONCLUSION}

Nonholonomic constraints of mechanical systems result in an additional reduction of the dimension of the dynamical system under consideration. If, in addition, the applied forces follow from a potential, a dynamical system with symmetry is given which means a further reduction of the Riemannian space representing the system's motion. It is shown that a nonlinearily controlled nonholonomic robot features full controllability operating on a rigid surface even with one control input.

\section{REFERENCES}

Bahar, L. Y. (1993). On a nonholonomic problem proposed by Greenwood, Int. J. Nonlinear Mechanics 28 , pp. 169-186.

Bianchi, G.; Schiehlen, W. (eds.) (1986). Dynamics of Multibody Systems, Springer-Verlag, Berlin.

Haug, E. J. (ed.) (1984). Computer-aided Analysis and Optimization of Mechanical System Dynamics, Springer-Verlag, Berlin.

Haug, E. J. (1989). Computer-aided Kinematics and Dynamics of Mechanical Systems, Allyn and Bacon, Boston.

Haug, E. J. (ed.) (1993). Concurrent Engineering: Tools and Technologies for Mechanical System Design, Springer-Verlag, Berlin.

Kane, T. R. (1978). Nonholonomic Multibody Systems Containing Gyrostats, in Dynamics of Multibody Systems (Ed. Magnus, K.), pp. 97-107, Springer-Verlag, Berlin.

Kortüm, W.; Sharp, R. S. (eds.) (1993). Multibody Computer Codes in Vehicle System Dynamics, Swets and Zeitlinger, Amsterdam.

Krishnaprasad, P. S.; Yang, R. and Dayawansa, W. P. (1991). Control Problems an Principal Bundles and Nonholonomic Mechanics, in Proc. 30th
IEEE Conference Decision and Control, pp. 1133-1138.

Magnus, K. (ed.) (1978). Dynamics of Multibody Systems. Springer-Verlag, Berlin.

Marsden, J. E.; Montgomery, R. and Ratiu, T. (1990). Reduction, Symmetry and Phases in Mechanics. Memoirs of the American Mathematical Society.

Neimark, J. I. and Futaev, N. A. (1972). Dynamics of Nonholonomic Systems, Translations of Mathematical Monographs, Vol. 33, American Mathematical Society, Providence.

Nikravesh, P. E. (1988). Computer-aided Analysis of Mechanical Systems, Prentice-Hall, New Jersey.

Roberson, R. E. and Schwertassek, R. (1988). Dynamics of Multibody Systems, Springer-Verlag, Berlin.

Schiehlen, W. (1977). Bewegungsverhalten eines nichtlinearen, nichtholonomen Systems, in VII. Int. Konferenz über nichtlineare Schwingungen, pp. 331-340, Band II,2, Akademie-Verlag, Berlin.

Schiehlen, W. (1986). Technische Dynamik, Stuttgart.

Schiehlen, W. (ed.) (1990). Multibody System Handbook, Springer-Verlag, Berlin.

Schiehlen, W. (1993). Nonlinear Oscillation in Multibody Systems, , in 1st European Nonlinear Oscillation Conf., pp. 85-106, Akademic-Verlag, Berlin.

Shabana, A. (1989). Dynamics of Multibody Systems, Wiley, New York.

Wittenburg, J. (1977). Dynamics of Systems of Rigid Bodies, B. G. Teubner, Stuttgart.

Yang, R. (1992). Nonholonomic Geometry, Mechanics and Control, Ph. D. Theses, University of Maryland, College Park.

Zexiang Li and J. F. Canny (1993). Nonholonomic Motion Planning, Kluwer, Dordrecht. 